\title{
Existence of nonnegative solutions for a fractional m-point boundary value problem at resonance
}

Haidong Qu ${ }^{1 *}$ and Xuan Liu²

\section{"Correspondence:}

qhaidong@163.com

1 Department of Mathematics,

Hanshan Normal University,

Chaozhou, Guangdong 521041,

China

Full list of author information is

available at the end of the article

\section{Abstract}

We consider the fractional differential equation

$$
D_{0+}^{q} u(t)=f(t, u(t)), \quad 0<t<1
$$

satisfying the boundary conditions

$$
\left.D_{0+}^{p} u(t)\right|_{t=0}=\left.D_{0+}^{p-1} u(t)\right|_{t=0}=\cdots=\left.D_{0+}^{p-n+1} u(t)\right|_{t=0}=0, \quad u(1)=\sum_{i=1}^{m-2} \alpha_{i} u\left(\xi_{i}\right),
$$

where $D_{0+}^{q}$ is the Riemann-Liouville fractional order derivative. The parameters in the multi-point boundary conditions are such that the corresponding differential operator is a Fredholm map of index zero. As a result, the minimal and maximal nonnegative solutions for the problem are obtained by using a fixed point theorem of increasing operators.

MSC: $26 \mathrm{~A} 33 ; 34 \mathrm{~A} 08$

Keywords: fractional order; coincidence degree; at resonance

\section{Introduction}

Let us consider the fractional differential equation

$$
D_{0+}^{q} u(t)=f(t, u(t)), \quad 0<t<1,
$$

with the boundary conditions (BCs)

$$
\left\{\begin{array}{l}
\left.D_{0+}^{p} u(t)\right|_{t=0}=\left.D_{0+}^{p-1} u(t)\right|_{t=0}=\cdots=\left.D_{0+}^{p-n+1} u(t)\right|_{t=0}=0, \\
u(1)=\sum_{i=1}^{m-2} \alpha_{i} u\left(\xi_{i}\right),
\end{array}\right.
$$

where $n \geq 1, \max \{q-2,0\} \leq p<q-1, n<q \leq n+1, \sum_{i=1}^{m-2} \alpha_{i} \xi_{i}^{q-1}=1, \alpha_{i}>0,0<\xi_{1}<$ $\xi_{2}<\cdots<\xi_{m-2}<1, m \geq 3$. We assume that $f:[0,1] \times[0, \infty) \rightarrow[0, \infty)$ is continuous. A boundary value problem at resonance for ordinary or fractional differential equations has been studied by several authors, including the most recent works [1-7] and the references therein. In the most papers mentioned above, the coincidence degree theory was

\section{Springer}

(c) 2013 Qu and Liu; licensee Springer. This is an Open Access article distributed under the terms of the Creative Commons Attribution License (http://creativecommons.org/licenses/by/2.0), which permits unrestricted use, distribution, and reproduction in any medium, provided the original work is properly cited. 
applied to establish existence theorems. But in [8], Wang obtained the minimal and maximal nonnegative solutions for a second-order m-point boundary value problem at resonance by using a new fixed point theorem of increasing operators, and in this paper we use this method of Wang to establish the existence theorem of equations (1.1) and (1.2).

For the convenience of the reader, we briefly recall some notations.

Let $X, Z$ be real Banach spaces, $L: \operatorname{dom}(L) \subset X \rightarrow Z$ be a Fredholm map of index zero and $P: X \rightarrow X, Q: Z \rightarrow Z$ be continuous projectors such that $\operatorname{Im}(P)=\operatorname{Ker}(L), \operatorname{Ker}(Q)=$ $\operatorname{Im}(L)$ and $X=\operatorname{Ker}(L) \oplus \operatorname{Ker}(P), Z=\operatorname{Im}(L) \oplus \operatorname{Im}(Q)$. It follows that $\left.L\right|_{\operatorname{Ker}(P) \cap \operatorname{dom}(L)}: \operatorname{Ker}(P) \cap$ $\operatorname{dom}(L) \rightarrow \operatorname{Im}(L)$ is invertible. We denote the inverse of the map by $K_{P}: \operatorname{Im}(L) \rightarrow \operatorname{Ker}(P) \cap$ $\operatorname{dom}(L)$. Since $\operatorname{dim} \operatorname{Im}(Q)=\operatorname{dim} \operatorname{Ker}(L)$, there exists an isomorphism $J: \operatorname{Im}(Q) \rightarrow \operatorname{Ker}(L)$. Let $\Omega$ be an open bounded subset of $X$. The map $N: X \rightarrow Z$ will be called $L$-compact on $\bar{\Omega}$ if $Q N(\bar{\Omega})$ and $K_{P}(I-Q)(\bar{\Omega})$ are compact. We take $H=L+J^{-1} P$, then $H: \operatorname{dom}(L) \subset X \rightarrow Z$ is a linear bijection with bounded inverse and $\left(J Q+K_{P}(I-Q)\right)\left(L+J^{-1} P\right)=\left(L+J^{-1} P\right)(J Q+$ $\left.K_{P}(I-Q)\right)=I$. We know from [9] that $K_{1}=H(K \cap \operatorname{dom}(L))$ is a cone in $Z$.

Theorem 1.1 [9] $N(u)+J^{-1} P(u)=H(\tilde{u})$, where

$$
\tilde{u}=P(u)+J Q N(u)+K_{P}(I-Q) N(u)
$$

and $\tilde{u}$ is uniquely determined.

From the above theorem, the author [9] obtained that the assertions

(i) $P(u)+J Q N(u)+K_{P}(I-Q) N(u): K \cap \operatorname{dom}(L) \rightarrow K \cap \operatorname{dom}(L)$ and

(ii) $N(u)+J^{-1} P(u): K \cap \operatorname{dom}(L) \rightarrow K_{1}$ are equivalent.

We also need the following definition and theorem.

Definition 1.1 [8] Let $K$ be a normal cone in a Banach space $X, u_{0} \leq v_{0}$, and $u_{0}, v_{0} \in$ $K \cap \operatorname{dom}(L)$ are said to be coupled lower and upper solutions of the equation $L x=N x$ if

$$
\left\{\begin{array}{l}
L u_{0} \leq N u_{0}, \\
L v_{0} \geq N v_{0} .
\end{array}\right.
$$

Theorem 1.2 [8] Let $L: \operatorname{dom}(L) \subset X \rightarrow Z$ be a Fredholm operator of index zero, $K$ be a normal cone in a Banach space $X, u_{0}, v_{0} \in K \cap \operatorname{dom}(L), u_{0} \leq v_{0}$, and $N:\left[u_{0}, v_{0}\right] \rightarrow Z$ be $L$-compact and continuous. Suppose that the following conditions are satisfied:

$\left(C_{1}\right) u_{0}$ and $v_{0}$ are coupled lower and upper solutions of the equation $L x=N x$;

$\left(C_{2}\right) N+J^{-1} P: K \cap \operatorname{dom}(L) \rightarrow K_{1}$ is an increasing operator.

Then the equation $L x=N x$ has a minimal solution $u^{*}$ and a maximal solution $v^{*}$ in $\left[u_{0}, v_{0}\right]$. Moreover,

$$
u^{*}=\lim _{n \rightarrow \infty} u_{n}, \quad v^{*}=\lim _{n \rightarrow \infty} v_{n}
$$

where

$$
u_{n}=\left(L+J^{-1} P\right)^{-1}\left(N+J^{-1} P\right) u_{n-1}, \quad v_{n}=\left(L+J^{-1} P\right)^{-1}\left(N+J^{-1} P\right) v_{n-1},
$$

$n=1,2,3, \ldots$ and $u_{0} \leq u_{1} \leq u_{2} \leq \cdots \leq u_{n} \leq \cdots \leq v_{n} \leq \cdots \leq v_{2} \leq v_{1} \leq v_{0}$. 


\section{Preliminaries}

In this section, we present some necessary basic knowledge and definitions about fractional calculus theory.

Definition 2.1 (see Equation 2.1.1 in [10]) The R-L fractional integral $I_{0+}^{q} u$ of order $q \in R$ $(q>0)$ is defined by

$$
I_{0+}^{q} u(t):=\frac{1}{\Gamma(q)} \int_{0}^{t} \frac{u(\tau) d \tau}{(t-\tau)^{1-q}} \quad(t>0)
$$

Here $\Gamma(q)$ is the gamma function.

Definition 2.2 (see Equation 2.1.5 in [10]) The R-L fractional derivative $D_{0+}^{q} u$ of order $q \in R(q>0)$ is defined by

$$
\begin{aligned}
D_{0+}^{q} u(t) & =\left(\frac{d}{d t}\right)^{n} I_{0+}^{n-q} u(t) \\
& =\frac{1}{\Gamma(n-q)}\left(\frac{d}{d t}\right)^{n} \int_{0}^{t} \frac{u(\tau) d \tau}{(t-\tau)^{q-n+1}} \quad(n=[q]+1, t>0),
\end{aligned}
$$

where $[q]$ means the integral part of $q$.

Lemma 2.1 [11] If $q_{1}, q_{2}>0, q>0$, then, for $u(t) \in L_{p}(0,1)$, the relations

$$
I_{0+}^{q_{1}} I_{0+}^{q_{2}} u(t)=I_{0+}^{q_{1}+q_{2}} u(t)
$$

and

$$
D_{0+}^{q_{1}} I_{0+}^{q_{1}} u(t)=u(t)
$$

hold a.e. on $[0,1]$.

Lemma 2.2 (see [11]) Let $q>0, n=[q]+1, D_{0+}^{q} u(t) \in L_{1}(0,1)$, then we have the equality

$$
I_{0+}^{q} D_{0+}^{q} u(t)=u(t)+\sum_{i=1}^{n} C_{i} t^{q-i}
$$

where $C_{i} \in R(i=1,2, \ldots, n)$ are some constants.

Lemma 2.3 (see Corollary 2.1 in [10]) Let $q>0$ and $n=[q]+1$, the equation $D_{0_{+}}^{q} u(t)=0$ is valid if and only if $u(t)=\sum_{i=1}^{n} C_{i} t^{q-i}$, where $C_{i} \in R(i=1,2, \ldots, n)$ are arbitrary constants.

Let $X=Z=C[0,1]$ with the norm $\|u\|=\sup _{t \in[0,1]}|u(t)|$, then $X$ and $Z$ are Banach spaces. Let $K=\{u \in X: u(t) \geq 0, t \in[0,1]\}$. It follows from Theorem 1.1.1 in [12] that $K$ is a normal cone.

Let $\operatorname{dom}(L)=\left\{u(t) \in X \mid D_{0_{+}}^{q} u(t) \in Z, u(t)\right.$ satisfies $\left.B C s(1.2)\right\}$.

We define the operators $L: \operatorname{dom}(L) \rightarrow Z$ by

$$
(L u)(t)=D_{0+}^{q} u(t)
$$


and $N: K \rightarrow Z$ by

$$
(N u)(t)=f(t, u(t))
$$

then BVPs (1.1) and (1.2) can be written as $L u=N u, u \in K \cap \operatorname{dom}(L)$.

Lemma 2.4 If the operator $L$ is defined in (2.1), then

(i) $\operatorname{Ker}(L)=\left\{c \cdot t^{q-1} \mid c \in R\right\}$,

(ii) $\operatorname{Im}(L)=\left\{y \in Z \mid \int_{0}^{1}(1-s)^{q-2} \sum_{i=1}^{m-2} \alpha_{i} \xi_{i}^{q-1} \int_{\xi_{i} s}^{s} y(\tau) d \tau d s=0\right\}=: \mathcal{L}$.

Proof (i) It can be seen from Lemma 2.3 and BCs (1.2) that $\operatorname{Ker}(L)=\left\{c \cdot t^{q-1} \mid c \in R\right\}$.

(ii) If $y \in \operatorname{Im}(L)$, then there exists a function $u \in \operatorname{dom}(L)$ such that $y(t)=D_{0_{+}}^{q} u(t)$, by Lemma 2.2, we have

$$
I_{0_{+}}^{q} y(t)=u(t)+c_{1} t^{q-1}+\cdots+c_{n} t^{q-n} .
$$

It follows from BCs (1.2) and the equation $\sum_{i=1}^{m-2} \alpha_{i} \xi_{i}^{q-1}=1$ that

$$
I_{0+}^{q} y(1)=\sum_{i=1}^{m-2} I_{0+}^{q} \alpha_{i} y\left(\xi_{i}\right)
$$

and noting the definition of $I_{0+}^{q}$, we have

$$
I_{0+}^{q} y(t)=\frac{1}{\Gamma(q)} \int_{0}^{t}(t-s)^{q-1} y(s) d s=\frac{q-1}{\Gamma(q)} \int_{0}^{t}(t-s)^{q-2} \int_{0}^{s} y(\tau) d \tau d s .
$$

Thus,

$$
\begin{aligned}
\frac{q-1}{\Gamma(q)} \int_{0}^{1}(1-s)^{q-2} \int_{0}^{s} y(\tau) d \tau d s & =\frac{q-1}{\Gamma(q)} \sum_{i=1}^{m-2} \alpha_{i} \int_{0}^{\xi_{i}}\left(\xi_{i}-s\right)^{q-2} \int_{0}^{s} y(\tau) d \tau d s \\
& =\frac{q-1}{\Gamma(q)} \sum_{i=1}^{m-2} \alpha_{i} \xi_{i} \int_{0}^{1}\left(\xi_{i}-\xi_{i} s\right)^{q-2} \int_{0}^{\xi_{i} s} y(\tau) d \tau d s \\
& =\frac{q-1}{\Gamma(q)} \sum_{i=1}^{m-2} \alpha_{i} \xi_{i}^{q-1} \int_{0}^{1}(1-s)^{q-2} \int_{0}^{\xi_{i} s} y(\tau) d \tau d s,
\end{aligned}
$$

which is

$$
\int_{0}^{1}(1-s)^{q-2} \sum_{i=1}^{m-2} \alpha_{i} \xi_{i}^{q-1} \int_{\xi_{i} s}^{s} y(\tau) d \tau d s=0 .
$$

Then $y \in \mathcal{L}$, hence $\operatorname{Im}(L) \subset \mathcal{L}$.

On the other hand, if $y \in \mathcal{L}$, let $u(t)=I_{0_{+}}^{q} y(t)$, then $u \in \operatorname{dom}(L)$, and $D_{0+}^{q} u(t)=D_{0+}^{q} I_{0+}^{q} y(t)=$ $y(t)$, which implies that $y \in \operatorname{Im}(L)$, thus $\mathcal{L} \subset \operatorname{Im}(L)$. In general $\operatorname{Im}(L)=\mathcal{L}$. Clearly, $\operatorname{Im}(L)$ is closed in $Z$ and $\operatorname{dim} \operatorname{Ker}(L)=\operatorname{codim} \operatorname{Im}(L)=1$, thus $L$ is a Fredholm operator of index zero. This completes the proof. 
In what follows, some property operators are defined. We define continuous projectors $P: X \rightarrow X$ by

$$
(P u)(t)=q \int_{0}^{1} u(s) d s \cdot t^{q-1}
$$

and $Q: Z \rightarrow Z$ by

$$
(Q u)(t)=\frac{1}{\gamma_{0}} \int_{0}^{1}(1-s)^{q-2} \sum_{i=1}^{m-2} \alpha_{i} \xi_{i}^{q-1} \int_{\xi_{i} s}^{s} u(\tau) d \tau d s,
$$

where

$$
\begin{aligned}
\gamma_{0} & =\int_{0}^{1}(1-s)^{q-2} \sum_{i=1}^{m-2} \alpha_{i} \xi_{i}^{q-1} \int_{\xi_{i} s}^{s} d \tau d s \\
& =\int_{0}^{1} s(1-s)^{q-2} d s\left(1-\sum_{i=1}^{m-2} \alpha_{i} \xi_{i}^{q}\right) \\
& =B(2, q-1)\left(1-\sum_{i=1}^{m-2} \alpha_{i} \xi_{i}^{q}\right)>0 .
\end{aligned}
$$

$B(x, y)$ is the beta function defined by

$$
B(x, y)=\int_{0}^{1} t^{x-1}(1-t)^{y-1} d t .
$$

By calculating, we easily obtain $P^{2}=P, Q^{2}=Q$, and $X=\operatorname{Ker}(L) \oplus \operatorname{Ker}(P), Z=\operatorname{Im}(L) \oplus$ $\operatorname{Im}(Q)$. We also define $J: \operatorname{Im}(Q) \rightarrow \operatorname{Ker}(L)$ by

$$
J(c)=c t^{q-1}, \quad \forall c \in R
$$

and $K_{P}: \operatorname{Im}(L) \rightarrow \operatorname{dom}(L) \cap \operatorname{Ker}(P)$ by

$$
\left(K_{P}(u)\right)(t)=\left(I_{0+}^{q} u\right)(t)=\frac{1}{\Gamma(q)} \int_{0}^{t}(t-s)^{q-1} u(s) d s,
$$

thus

$$
(Q N(u))(t)=\frac{1}{\gamma_{0}} \int_{0}^{1}(1-s)^{q-2} \sum_{i=1}^{m-2} \alpha_{i} \xi_{i}^{q-1} \int_{\xi_{i} s}^{s} f(\tau, u(\tau)) d \tau d s
$$

and

$$
\begin{aligned}
\left(K_{P}(I-Q) N(u)\right)(t) \\
=\frac{1}{\Gamma(q)} \int_{0}^{t}(t-s)^{q-1} f(s, u(s)) d s \\
\quad-\frac{1}{\Gamma(q)} \int_{0}^{t}(t-s)^{q-1} \frac{1}{\gamma_{0}} \int_{0}^{1}(1-\tilde{s})^{q-2} \sum_{i=1}^{m-2} \alpha_{i} \xi_{i}^{q-1} \int_{\xi_{i} \tilde{s}}^{\tilde{s}} f(\tau, u(\tau)) d \tau d \tilde{s} d s .
\end{aligned}
$$


Lemma 2.5 Let $\Omega$ be any open bounded subset of $K \cap \operatorname{dom}(L)$, then $Q N(\bar{\Omega})$ and $K_{P}(I-$ Q)N $(\bar{\Omega})$ are compact, which implies that $N$ is L-compact on $\bar{\Omega}$ for any open bounded set $\Omega \subset K \cap \operatorname{dom}(L)$.

Proof For a positive integer $n$, let $\Omega=\{u \in K \cap \operatorname{dom}(L):\|u\| \leq n\}, M=\sup _{(t, u)} f(t, u(t))$, $(t, u) \in[0,1] \times[0, n]$. It is easy to see that $Q N(\bar{\Omega})$ is compact. Now, we prove that $K_{P}(I-$ Q) $N(\bar{\Omega})$ is compact. For $\forall u \in \bar{\Omega}$, we have

$$
\begin{aligned}
\| & \left(K_{P}(I-Q) N(u)\right)(t) \| \\
= & \sup _{t \in[0,1]} \mid \frac{1}{\Gamma(q)} \int_{0}^{t}(t-s)^{q-1} f(s, u(s)) d s \\
& \quad-\frac{1}{\Gamma(q)} \int_{0}^{t}(t-s)^{q-1} \frac{1}{\gamma_{0}} \int_{0}^{1}(1-\tilde{s})^{q-2} \sum_{i=1}^{m-2} \alpha_{i} \xi_{i}^{q-1} \int_{\xi_{i} \tilde{s}}^{\tilde{s}} f(\tau, u(\tau)) d \tau d \tilde{s} d s \mid \\
\leq & \sup _{t \in[0,1]}\left|\frac{1}{\Gamma(q)} \int_{0}^{t}(t-s)^{q-1} f(s, u(s)) d s\right| \\
& +\sup _{t \in[0,1]}\left|\frac{1}{\Gamma(q)} \int_{0}^{t}(t-s)^{q-1} \frac{1}{\gamma_{0}} \int_{0}^{1}(1-\tilde{s})^{q-2} \sum_{i=1}^{m-2} \alpha_{i} \xi_{i}^{q-1} \int_{\xi_{i} \tilde{s}}^{\tilde{s}} f(\tau, u(\tau)) d \tau d \tilde{s} d s\right| \\
\leq & \frac{2 M}{\Gamma(q)} \sup _{t \in[0,1]}\left|\int_{0}^{t}(t-s)^{q-1} d s\right| \\
= & \frac{2 M}{\Gamma(q+1)},
\end{aligned}
$$

which implies that $K_{P}(I-Q) N(\bar{\Omega})$ is bounded.

Moreover, for each $u \in \bar{\Omega}$, let $t_{1}, t_{2} \in[0,1]$ and $t_{1}>t_{2}$, then

$$
\begin{aligned}
\|( & \left.K_{P}(I-Q) N(u)\right)\left(t_{1}\right)-\left(K_{P}(I-Q) N(u)\right)\left(t_{2}\right) \| \\
\leq & \left|\frac{1}{\Gamma(q)} \int_{0}^{t_{1}}\left(t_{1}-s\right)^{q-1} f(s, u(s)) d s-\frac{1}{\Gamma(q)} \int_{0}^{t_{2}}\left(t_{2}-s\right)^{q-1} f(s, u(s)) d s\right| \\
& +\mid \frac{1}{\Gamma(q)} \int_{0}^{t_{1}}\left(t_{1}-s\right)^{q-1} \frac{1}{\gamma_{0}} \int_{0}^{1}(1-\tilde{s})^{q-2} \sum_{i=1}^{m-2} \alpha_{i} \xi_{i}^{q-1} \int_{\xi_{i} \tilde{s}}^{\tilde{s}} f(\tau, u(\tau)) d \tau d \tilde{s} d s \\
& -\frac{1}{\Gamma(q)} \int_{0}^{t_{2}}\left(t_{2}-s\right)^{q-1} \frac{1}{\gamma_{0}} \int_{0}^{1}(1-\tilde{s})^{q-2} \sum_{i=1}^{m-2} \alpha_{i} \xi_{i}^{q-1} \int_{\xi_{i} \tilde{s}}^{\tilde{s}} f(\tau, u(\tau)) d \tau d \tilde{s} d s \mid \\
\leq & \left|\frac{1}{\Gamma(q)} \int_{0}^{t_{2}}\left(t_{1}-s\right)^{q-1} f(s, u(s)) d s-\frac{1}{\Gamma(q)} \int_{0}^{t_{2}}\left(t_{2}-s\right)^{q-1} f(s, u(s)) d s\right| \\
& +\left|\frac{1}{\Gamma(q)} \int_{t_{2}}^{t_{1}}\left(t_{1}-s\right)^{q-1} f(s, u(s)) d s\right| \\
+ & \frac{1}{\Gamma(q)} \int_{0}^{t_{2}}\left(t_{1}-s\right)^{q-1} \frac{1}{\gamma_{0}} \int_{0}^{1}(1-\tilde{s})^{q-2} \sum_{i=1}^{m-2} \alpha_{i} \xi_{i}^{q-1} \int_{\xi_{i} \tilde{s}}^{\tilde{s}} f(\tau, u(\tau)) d \tau d \tilde{s} d s \\
& \quad-\frac{1}{\Gamma(q)} \int_{0}^{t_{2}}\left(t_{2}-s\right)^{q-1} \frac{1}{\gamma_{0}} \int_{0}^{1}(1-\tilde{s})^{q-2} \sum_{i=1}^{m-2} \alpha_{i} \xi_{i}^{q-1} \int_{\xi_{i} \tilde{s}}^{\tilde{s}} f(\tau, u(\tau)) d \tau d \tilde{s} d s \mid
\end{aligned}
$$




$$
\begin{aligned}
& +\left|\frac{1}{\Gamma(q)} \int_{t_{2}}^{t_{1}}\left(t_{1}-s\right)^{q-1} \frac{1}{\gamma_{0}} \int_{0}^{1}(1-\tilde{s})^{q-2} \sum_{i=1}^{m-2} \alpha_{i} \xi_{i}^{q-1} \int_{\xi_{i} \tilde{s}}^{\tilde{s}} f(\tau, u(\tau)) d \tau d \tilde{s} d s\right| \\
\leq & \frac{2 M}{\Gamma(q)}\left|\int_{0}^{t_{1}}\left(t_{1}-s\right)^{q-1} d s-\int_{0}^{t_{2}}\left(t_{2}-s\right)^{q-1} d s\right|+\frac{2 M}{\Gamma(q)}\left|\int_{t_{2}}^{t_{1}}\left(t_{1}-s\right)^{q-1} d s\right| \\
\leq & \frac{2 M}{\Gamma(q)}\left|\int_{0}^{t_{1}}\left(t_{1}-s\right)^{q-1} d s-\int_{0}^{t_{2}}\left(t_{2}-s\right)^{q-1} d s\right|+\frac{2 M}{\Gamma(q)}\left|t_{1}-t_{2}\right| \\
= & \frac{2 M}{\Gamma(q)}\left|t_{1} \int_{0}^{1}\left(t_{1}-t_{1} s\right)^{q-1} d s-t_{2} \int_{0}^{1}\left(t_{2}-t_{2} s\right)^{q-1} d s\right|+\frac{2 M}{\Gamma(q)}\left|t_{1}-t_{2}\right| \\
= & \frac{2 M}{\Gamma(q+1)}\left|t_{1}^{q}-t_{2}^{q}\right|+\frac{2 M}{\Gamma(q)}\left|t_{1}-t_{2}\right| \\
= & \frac{2 M}{\Gamma(q+1)}\left|q \eta^{q-1}\right| \cdot\left|t_{1}-t_{2}\right|+\frac{2 M}{\Gamma(q)}\left|t_{1}-t_{2}\right|, \quad \eta=t_{1}+\theta\left(t_{2}-t_{1}\right), 0<\theta<1 \\
\leq & \frac{\left(2^{q}+2\right) M}{\Gamma(q)}\left|t_{1}-t_{2}\right| .
\end{aligned}
$$

Thus

$$
\forall \varepsilon>0, \quad \exists \delta=\frac{\Gamma(q)}{\left(2^{q}+2\right) M} \varepsilon
$$

such that

$$
\left\|K_{P}(I-Q) N(u)\left(t_{1}\right)-K_{P}(I-Q) N(u)\left(t_{2}\right)\right\|<\varepsilon
$$

for

$$
\left|t_{1}-t_{2}\right|<\delta
$$

and each

$$
u \in \bar{\Omega}
$$

It is concluded that $N$ is $L$-compact on $\bar{\Omega}$. This completes the proof.

\section{Main result}

In this section, we establish the existence of the nonnegative solution to equations (1.1) and (1.2).

\section{Theorem 3.1 Suppose}

$\left(\mathrm{H}_{1}\right)$ There exist $u_{0}, v_{0} \in K \cap \operatorname{dom}(L)$ such that $u_{0} \leq v_{0}$ and

$$
\begin{cases}D_{0+}^{q} u_{0}(t) \leq f\left(t, u_{0}(t)\right), & \forall t \in[0,1], \\ D_{0+}^{q} v_{0}(t) \geq f\left(t, v_{0}(t)\right), & \forall t \in[0,1] .\end{cases}
$$

$\left(\mathrm{H}_{2}\right)$ For any $x, y \in K \cap \operatorname{dom}(L)$, satisfying

$$
f(t, x(t))-f(t, y(t)) \geq-q\left(\int_{0}^{1} x(t) d t-\int_{0}^{1} y(t) d t\right)
$$


where $\forall t \in[0,1]$ and $u_{0}(t) \leq y(t) \leq x(t) \leq v_{0}(t)$, then problems (1.1) and (1.2) have a minimal solution $u^{*}$ and a maximal solution $v^{*}$ in $\left[u_{0}, v_{0}\right]$, respectively.

Proof By condition $\left(\mathrm{H}_{1}\right)$, we know that

$$
L u_{0} \leq N u_{0}, \quad L v_{0} \geq N v_{0}
$$

so condition $\left(\mathrm{C}_{1}\right)$ in Theorem 1.1 holds.

In addition, for each $u \in K$,

$$
\begin{aligned}
(P(u) & \left.+J Q N(u)+K_{P}(I-Q) N(u)\right)(t) \\
=q & \int_{0}^{1} u(s) d s \cdot t^{q-1}+\frac{1}{\gamma_{0}} \int_{0}^{1}(1-s)^{q-2} \sum_{i=1}^{m-2} \alpha_{i} \xi_{i}^{q-1} \int_{\xi_{i} s}^{s} f(\tau, u(\tau)) d \tau d s \cdot t^{q-1} \\
& +\frac{1}{\Gamma(q)} \int_{0}^{t}(t-s)^{q-1} f(s, u(s)) d s \\
& -\frac{1}{\Gamma(q)} \int_{0}^{t}(t-s)^{q-1} \frac{1}{\gamma_{0}} \int_{0}^{1}(1-\tilde{s})^{q-2} \sum_{i=1}^{m-2} \alpha_{i} \xi_{i}^{q-1} \int_{\xi_{i} \tilde{s}}^{\tilde{s}} f(\tau, u(\tau)) d \tau d \tilde{s} d s \\
= & q \int_{0}^{1} u(s) d s \cdot t^{q-1}+\frac{1}{\Gamma(q)} \int_{0}^{t}(t-s)^{q-1} f(s, u(s)) d s \\
& +\frac{1}{\gamma_{0}} \int_{0}^{1}(1-s)^{q-2} \sum_{i=1}^{m-2} \alpha_{i} \xi_{i}^{q-1} \int_{\xi_{i} s}^{s} f(\tau, u(\tau)) d \tau d s\left(t^{q-1}-\frac{1}{\Gamma(q)} \int_{0}^{t}(t-s)^{q-1} d s\right) \\
\geq & \frac{1}{\gamma_{0}} \int_{0}^{1}(1-s)^{q-2} \sum_{i=1}^{m-2} \alpha_{i} \xi_{i}^{q-1} \int_{\xi_{i} s}^{s} f(\tau, u(\tau)) d \tau d s\left(t^{q-1}-\frac{t^{q}}{\Gamma(q+1)}\right) \geq 0 .
\end{aligned}
$$

Thus $\left(P+J Q N+K_{P}(I-Q) N\right)(K) \subset K$, that is, $N+J^{-1} P: K \cap \operatorname{dom}(L) \rightarrow K_{1}$ by virtue of the equivalence. From condition $\left(\mathrm{H}_{2}\right)$, we have that $N+J^{-1} P: K \cap \operatorname{dom}(L) \rightarrow K_{1}$ is a monotone increasing operator. Then, in accordance with Lemma 2.5 and Theorem 1.2, we obtain a minimal solution $u^{*}$ and a maximal solution $v^{*}$ in $\left[u_{0}, v_{0}\right]$ for problems (1.1) and (1.2). Thus we can define iterative sequences $\left\{u_{n}(t)\right\}$ and $\left\{v_{n}(t)\right\}$ by

$$
\begin{aligned}
u_{n}= & \left(L+J^{-1} P\right)^{-1}\left(N+J^{-1} P\right) u_{n-1}=\left(J Q+K_{P}(I-Q)\right)\left(N+J^{-1} P\right) u_{n-1} \\
= & \left(J Q+K_{P}(I-Q)\right)\left(f\left(t, u_{n-1}(t)\right)+q \int_{0}^{1} u_{n-1}(s) d s\right) \\
= & \frac{1}{\gamma_{0}} \int_{0}^{1}(1-s)^{q-2} \sum_{i=1}^{m-2} \alpha_{i} \xi_{i}^{q-1} \int_{\xi_{i} s}^{s}\left(f\left(\tau, u_{n-1}(\tau)\right)+q \int_{0}^{1} u_{n-1}(\hat{s}) d \hat{s}\right) d \tau d s \cdot t^{q-1} \\
& +\frac{1}{\Gamma(q)} \int_{0}^{t}(t-s)^{q-1}\left(f\left(s, u_{n-1}(s)\right)+q \int_{0}^{1} u_{n-1}(\tilde{s}) d \tilde{s}\right) d s \\
& -\frac{1}{\Gamma(q)} \int_{0}^{t}(t-s)^{q-1} \frac{1}{\gamma_{0}} \int_{0}^{1}(1-\tilde{s})^{q-2} \sum_{i=1}^{m-2} \alpha_{i} \xi_{i}^{q-1} \\
& \cdot \int_{\xi_{i} \tilde{s}}^{\tilde{s}}\left(f\left(\tau, u_{n-1}(\tau)\right)+q \int_{0}^{1} u_{n-1}(\hat{s}) d \hat{s}\right) d \tau d \tilde{s} d s
\end{aligned}
$$


and

$$
\begin{aligned}
v_{n}= & \left(L+J^{-1} P\right)^{-1}\left(N+J^{-1} P\right) v_{n-1}=\left(J Q+K_{P}(I-Q)\right)\left(N+J^{-1} P\right) v_{n-1} \\
= & \left(J Q+K_{P}(I-Q)\right)\left(f\left(t, v_{n-1}(t)\right)+q \int_{0}^{1} v_{n-1}(s) d s\right) \\
= & \frac{1}{\gamma_{0}} \int_{0}^{1}(1-s)^{q-2} \sum_{i=1}^{m-2} \alpha_{i} \xi_{i}^{q-1} \int_{\xi_{i} s}^{s}\left(f\left(\tau, v_{n-1}(\tau)\right)+q \int_{0}^{1} v_{n-1}(\hat{s}) d \hat{s}\right) d \tau d s \cdot t^{q-1} \\
& +\frac{1}{\Gamma(q)} \int_{0}^{t}(t-s)^{q-1}\left(f\left(s, v_{n-1}(s)\right)+q \int_{0}^{1} v_{n-1}(\tilde{s}) d \tilde{s}\right) d s \\
& -\frac{1}{\Gamma(q)} \int_{0}^{t}(t-s)^{q-1} \frac{1}{\gamma_{0}} \int_{0}^{1}(1-\tilde{s})^{q-2} \sum_{i=1}^{m-2} \alpha_{i} \xi_{i}^{q-1} \\
& \cdot \int_{\xi_{i} \tilde{s}}^{\tilde{s}}\left(f\left(\tau, v_{n-1}(\tau)\right)+q \int_{0}^{1} v_{n-1}(\hat{s}) d \hat{s}\right) d \tau d \tilde{s} d s, \quad n=1,2,3, \ldots
\end{aligned}
$$

Then from Theorem 1.2 we get $\left\{u_{n}\right\}$ and $\left\{v_{n}\right\}$ converge uniformly to $u^{*}(t)$ and $v^{*}(t)$, respectively. Moreover,

$$
u_{0} \leq u_{1} \leq u_{2} \leq \cdots \leq u_{n} \leq \cdots \leq v_{n} \leq \cdots \leq v_{2} \leq v_{1} \leq v_{0}
$$

\section{Example}

We consider the following problem:

$$
D_{0+}^{\frac{3}{2}} u(t)=\left(\frac{u^{2}}{u^{2}+1}+t\right)^{m}, \quad 0<t<1, m>0
$$

subject to BCs

$$
\left.D_{0+}^{\frac{1}{4}} u(t)\right|_{t=0}=0, \quad u(1)=\sqrt{2} u\left(\frac{1}{2}\right) .
$$

We can choose

$$
u_{0}(t)=\frac{1}{\Gamma\left(\frac{3}{2}\right)} \int_{0}^{t}(t-s)^{\frac{1}{2}} s^{m} d s+t^{\frac{1}{2}} \leq \frac{1}{\Gamma\left(\frac{3}{2}\right)} \int_{0}^{t}(t-s)^{\frac{1}{2}}(s+1)^{m} d s+t^{\frac{1}{2}}=v_{0}(t)
$$

then

$$
D_{0+}^{\frac{3}{2}} u_{0}(t)=t^{m} \leq\left(\frac{u^{2}}{u^{2}+1}+t\right)^{m} \leq(t+1)^{m}=D_{0+}^{\frac{3}{2}} \nu_{0}(t)
$$

Let $\operatorname{dom}(L)=\left\{u(t) \in X \mid D_{0_{+}}^{\frac{3}{2}} u(t) \in Z, u(t)\right.$ satisfies BCs (4.2) $\}$, then for any $x, y \in K \cap$ $\operatorname{dom}(L)$, we have

$$
\left(\frac{x^{2}}{x^{2}+1}+t\right)^{m}-\left(\frac{y^{2}}{y^{2}+1}+t\right)^{m} \geq-\frac{3}{2}\left(\int_{0}^{1} x(t) d t-\int_{0}^{1} y(t) d t\right)
$$

where $u_{0}(t) \leq y(t) \leq x(t) \leq v_{0}(t)$. Finally, by Theorem 3.1, equation (4.1) with BCs (4.2) has a minimal solution $u^{*}$ and a maximal solution $v^{*}$ in $\left[u_{0}, v_{0}\right]$. 
Competing interests

The authors declare that they have no competing interests.

\section{Authors' contributions}

The authors declare that the study was realized in collaboration with the same responsibility. All authors read and approved the final manuscript.

\section{Author details}

${ }^{1}$ Department of Mathematics, Hanshan Normal University, Chaozhou, Guangdong 521041, China. ${ }^{2}$ Department of Basic Education, Hanshan Normal University, Chaozhou, Guangdong 521041, China.

\section{Acknowledgements}

The authors would like to thank the referees for their many constructive comments and suggestions to improve the paper

Received: 11 January 2013 Accepted: 26 April 2013 Published: 16 May 2013

\section{References}

1. Infantea, G, Zima, M: Positive solutions of multi-point boundary value problems at resonance. Nonlinear Anal. 69 , 2458-2465 (2008)

2. Kosmatov, N: Multi-point boundary value problems on an unbounded domain at resonance. Nonlinear Anal. 68 , 2158-2171 (2008)

3. Yang, L, Shen, CF: On the existence of positive solution for a kind of multi-point boundary value problem at resonance. Nonlinear Anal. 72, 4211-4220 (2010)

4. Bai, Z, Zhang, Y: The existence of solutions for a fractional multi-point boundary value problem. Comput. Math. Appl. 60, 2364-2372 (2010)

5. Zhang, Y, Bai, Z: Existence of solutions for nonlinear fractional three-point boundary value problems at resonance. J. Appl. Math. Comput. 36, 417-440 (2011)

6. Du, Z: Solvability of functional differential equations with multi-point boundary value problems at resonance. Comput. Math. Appl. 55, 2653-2661 (2008)

7. Han, X: Positive solutions for a three-point boundary value problem at resonance. J. Math. Anal. Appl. 36, 556-568 (2007)

8. Wang, F, Cui, YJ, Zhang, F: Existence of nonnegative solutions for second order m-point boundary value problems at resonance. Appl. Math. Comput. 217, 4849-4855 (2011)

9. Cremins, CT: A fixed-point index and existence theorems for semilinear equations in cones. Nonlinear Anal. 42 , 789-806 (2001)

10. Kilbsa, AA, Srivastava, HM, Trujillo, JJ: Theory and Applications of Fractional Differential Equations. Elsevier, Amsterdam (2006)

11. Chen, $Y$, Tang, $X$ : Positive solutions of fractional differential equations at resonance on the half-line. Bound. Value Probl. (2012). doi:10.1186/1687-2770-2012-64

12. Guo, DJ, Lakshmikantham, V: Nonlinear Problems in Abstract Cones. Academic Press, New York (1988)

doi:10.1186/1687-2770-2013-127

Cite this article as: Qu and Liu: Existence of nonnegative solutions for a fractional m-point boundary value problem at resonance. Boundary Value Problems 2013 2013:127.

\section{Submit your manuscript to a SpringerOpen ${ }^{\circ}$ journal and benefit from:}

- Convenient online submission

- Rigorous peer review

- Immediate publication on acceptance

- Open access: articles freely available online

- High visibility within the field

- Retaining the copyright to your article 